EESTI NSV TEADUSTE AKADEEMIA TOIMETISËD.

FUOSIKA * MATEMAATIKA

ИЗВЕСТИЯ АКАДЕМИИ НАУК ЭСТОНСКОИ ССР. ФИЗИКА * МАТЕМАТИКА

PROCEEDINGS OF THE ACADEMY OF SCIENCES OF THE ESTONIAN SSR.

PHYSICS * MATHEMATICS

$1988,37,1$

удК 517.977 .58

P. TEHHO

\title{
АППРОКСИМАЦИЯ ОПТИМАЛЬНЫХ УПРАВЛЕНИЙ БИЛИНЕЙНЫ ДИФФУЗИОННЫМ ПРОЦЕССОМ
}

R. TENNO, BILINEAARSE DIFUSIOONIPROTSESSI OPTIMAALJUHTIMISE APROKSIMATSIOON

R. TENNO. APPROXIMATION FOR BILINEAR QUADRATIC STOCHASTIC CONTROL PROBLEM

$$
\text { (Представил Н. Алумяэ) }
$$

Предложен вероятностный метод приближенного решения уравнения Беллмана для задачи оптимального управления билинейным диффузионным процессом, наблюдаемым вместе с погрешностями измерений.

\section{1. Постановка задачи}

Ставится задача оптимального управления - минимизации квадратичного функционала

$$
v^{\alpha}=M\left\{\int_{0}^{T}\left(\Theta_{t}^{2}+\alpha_{t}^{\tau} \Upsilon \alpha_{t}\right) d t+\Theta_{T}^{2}\right\},
$$

относительно частично наблюдаемого случайного процесса с дифференциалом

$$
\begin{aligned}
d \Theta=\left[g\left(\alpha_{t}\right) \Theta_{t}+\alpha_{t}^{r} a_{0}\right] \mathrm{dt}+d W, & \Theta_{0}=\tilde{\Theta}, \\
d \xi=\left[A(t, \xi)+C \Theta_{t}\right] \mathrm{dt}+d V, & \xi_{0}=0 .
\end{aligned}
$$

Здесь $\alpha$ - вектор управлений, $\Upsilon$ - положительно определенная матрица, $\left(\Theta_{t}\right)$ - ненаблюдаемый одномерный процесс, $g(\alpha)$ - линейная функция

$$
g(\alpha)=a_{1}+\alpha^{r} a_{2}
$$

$\tilde{\Theta}$ - гауссовое начальное значение; $(\xi t)$ - наблюдаемый векторный процесс, $A(t, \cdot)$ - функция, удовлетворяющая условиям линейного роста и Липшица, $\left(W_{t}\right)$ и $\left(V_{t}\right)$ - независимые винеровские процессы.

Допустимые управления заданы всеми неупреждающими функциями $\boldsymbol{\alpha}(t, \xi)$, такими, при которых система уравнений $(2)$ имеет слабое решение, например, всеми неупреждающими ограниченными функциями. В этих случаях стоимость управления $v^{\alpha}$ корректно определена [ $\left.{ }^{1}\right]$.

\section{2. Оптимальное управление}

C помощью уравнений оптимальной нелинейной фильтрации $\left[{ }^{2}\right]$ задачу управления по неполным данным (1), (2) можно свести к полным, т. е. к задаче минимизации функционала 


$$
v^{\alpha}=M\left\{\int_{0}^{T}\left(m_{t}^{2}+\gamma_{t}+\alpha_{t}^{T} \Upsilon \alpha_{t}\right) d t+m_{T}^{2}+\gamma_{T}\right\}
$$

относительно фильтрационного процесса $\left(m_{t}, \gamma_{t}\right)$ с уравнениями

$$
\begin{array}{rlrl}
d m & =\left[g(\alpha) m_{t}+\alpha_{t}^{r} a_{0}\right] \mathrm{dt}+\gamma_{t} C^{r} d \bar{W}, & m_{0}=m_{0}, \\
\dot{\gamma} & =2 g(\alpha) \gamma_{t}+1-\gamma_{t}^{2} C^{r} C, & \gamma_{0} & =\gamma_{0},
\end{array}
$$

где $\left(\bar{W}_{t}\right)$ - винеровский процесс с дифференциалом

$$
d \bar{W}=d \xi-\left[A(t, \xi)+C m_{t}\right] d t .
$$

Предположим, что существуют все производные, которые входят в уравнения Беллмана для задачи (3), (4), и что это уравнение имеет единственное решение *. Если оптимальное управление существует **, то из уравнения Беллмана нетрудно вывести, что оно задается равенством

$$
\alpha_{t}^{*}=-\Upsilon^{-1} L(v),
$$

где

$$
L(v)=\frac{1}{2}\left(a_{0}+a_{2} m\right) \frac{\partial v}{\partial m}+a_{2} \gamma \frac{\partial v}{\partial \gamma}
$$

$v=v\left(m_{t}, \gamma_{t}\right)$ - решение дифференциального уравнения

$$
\frac{\gamma^{2}}{2} C^{r} C \frac{\partial^{2} v}{\partial m^{2}}+a_{1} m \frac{\partial v}{\partial m}+\left[2 a_{1} \gamma+1-\gamma^{2} C^{r} C\right] \frac{\partial v}{\partial \gamma}+m^{2}+\gamma-L^{r} \Upsilon^{-1} L=0
$$

с граничным условием

$$
v\left(m_{T}, \gamma_{T}\right)=m_{T}^{2}+\gamma_{T}
$$

Ввиду сложности уравнение (6) не может быть решено аналитическим методом. Численным методом конечных разностей [ $\left.{ }^{3}\right]$ можно найти его приближенное решение в случае одномерного управления. Однако без сглаживания это численное решение не может быть применено для вычисления производных функций $v\left(m_{t}, \gamma_{t}\right)$, а тем самым и для вычисления управлений (5). Сглаживание требует подробного анализа результатов вычислений в каждом случае. Оценивание вида и параметров функции $v\left(m_{t}, \gamma_{t}\right)$ по дискретным расчетным (неточным) значениям дело не простое.

Приведем другой, более удобный метод приближенного решения уравнения Беллмана (6) и аппроксимации оптимальных управлений (5).

\section{3. Метод аппроксимации}

Пусть $\Delta t>0$ и $T / \Delta t=N-$ целое число. Определим последовательность $\left(m_{t}, \gamma_{t}, t=0, \Delta t, \ldots, N \Delta t\right) \quad$ с уравнениями

$$
\begin{aligned}
& m_{t+\Delta t}=m_{t}+\left[g(\alpha) m_{t}+\alpha_{t}^{\tau} a_{0}\right] \Delta t+\sigma \sqrt{\Delta t} \varepsilon_{t+\Delta t}, \\
& \gamma_{t+\Delta t}=(1+g(\alpha) \Delta t)^{2} \gamma_{t}+\Delta t-\sigma^{2} \Delta t,
\end{aligned}
$$

* Уравнение Беллмана имеет единственное решение (см. теоремы 7.7 .4 и 14.3 .5 в [1]) в случае приближення управляемого процесса (4) к невырождающимся. ** В условии выпуклости множества допустимых управлений существует $\varepsilon$-оптимальное управление (см. лемму 1.1.5 в [1]). 
где $\left(\varepsilon_{t}\right)$ - об̆новляющаa последовательность из неза̇висимых нормиро́ванных гауссовых величин, $\sigma$ - усиление фильтра

$$
\sigma=\frac{1+g(\alpha) \Delta t}{\sqrt{1+C^{T} \gamma C \Delta t}} \gamma C^{T}
$$

и функционал

$$
v_{N}^{\alpha}=M\left\{\sum_{t=0}^{(N-1) \Delta t}\left(m_{t}^{2}+\gamma_{t}+\alpha_{t}^{r} \Upsilon \alpha_{t}\right) \Delta t+m_{N \Delta t}^{2}+\gamma_{N \Delta t}\right\} .
$$

Сформулируем задачу управления последовательностью, как задачу определения таких управлений $\left(\alpha_{t}\right)$, которые минимизируют функционал (8) относительно системы (7). вый

Данная задача является аппроксимационной ввиду следующего. Пер-

$$
M\left(m_{t+\Delta t}-m_{t} / m_{t}=m\right)=\left[g(\alpha) m+\alpha^{r} a_{0}\right] \Delta t
$$

и второй момент

$$
\operatorname{var}\left(m_{t+\Delta t}-m_{t} / m_{t}=m\right)=\sigma^{2} \Delta t
$$

условного (гауссового) распределения $P\left(m_{t+\Delta t}-m_{t} / m_{t}=m\right)$ состоятельны (при $\Delta t \rightarrow 0$ ) относительно соответствующих моментов диффузионного процесса $\left(m_{t}\right)$ с дифференциалом (4). Тем самым ступенчатый процесс, построенный путем интерполяции последовательности (7), сходится к случайному процессу с дифференциалом (4) в слабом смысле, т. е. в смысле мер, а оптимальная стоимость управления последовательностью

$$
v_{N}=\inf v_{N}^{\alpha}
$$

к оптимальной стоимости управления случайным процессом (см. теорему 3.8 в $\left.\left[{ }^{4}\right]\right)$

$$
v=\inf v^{\alpha} \text {. }
$$

Если $N=3$, то задача (7), (8) поддается точному аналитическому решению [ $\left.{ }^{5}\right]$. Оптимальная стоимость $v_{3}$ выражается через начальные условия $\gamma_{0}, m_{0}$ уравнений фильтраций (7). Если в качестве начальных условий принимать текущие (в зависимости $\left.v_{3}\left(m_{0}, \gamma_{0}\right)\right)$, то стоимость $v_{3}\left(m_{t}, \gamma_{t}\right)$ аппроксимирует стоимость, оптимальную управлению последовательностью в текущий момент времени

$$
v_{N}\left(m_{t}, \gamma_{t}\right)=v_{3}\left(m_{t}, \gamma_{t}\right)+\mu_{t}+\varepsilon\left(m_{t}, \gamma_{t}\right)
$$

с точностью до неизвестного постоянного $\mu$ с малой погрешностью $\varepsilon$ $\left[{ }^{6}\right]$. А что особенно важно, $v_{3}\left(m_{t}, \gamma_{t}\right)$ аппроксимирует стоимость оптимального управления $v\left(m_{t}, \gamma_{t}\right)$ непрерывным процессом (4) в текущий момент времени или, что то же самое, аппроксимирует решение уравнения Беллмана (6) с некоторыми другими постоянным $\mu$ и погрешностью $\varepsilon$.

Оптимальное управление (5) от константы $\mu$ не зависит - оно зависит только от производных функции $v\left(m_{t}, \gamma_{t}\right)$. Для аппроксимации оптимального управления зависимостью типа (9) достаточно гладкости функции $\varepsilon\left(m_{t}, \gamma_{t}\right)$ по первым производным. Гладкость функции $\varepsilon\left(m_{t}, \gamma_{t}\right)$ подтверждается нашими результатами численного решения уравнения (6) и гладкостью функции $v_{2}\left(m_{t}, \gamma_{t}\right)\left[{ }^{5}\right]$. 


\section{ЛัИ Т Е А Т Р А}

1. Крылов Н. В. Управляемые процессы диффузионного типа. М., «Наука», 1977.

2. Липцер $P$. Ш., Ширяев А. Н. Статистика случайных процессов. Нелинейная фильтрация и смежные вопросы. М., «Наука», 1974.

3. Кушнер $\Gamma$. Дж. Вероятностные методы аппроксимации в стохастических задачах управления и теории эллиптических уравнений. М., «Наука», 1985.

4. Гихман И. И., Скороход А. В. Управляемые случайные процессы. Киев, «Наукова думка», 1977.

5. Тенно Р. Изв. АН ЭССР. Физ. Матем., 35, № 4, 435-436 (1986).

6. Тенно Р. Изв. АН ӘССР. Физ. Матем., 35, № 1, 62-70 (1986).

Ннститут кибернетики

Академии наук Эстонской ССР
Поступила в редакцию

10/VI 1987 